\title{
The Ranks of the Planarity Varieties of Commutative Semigroups
}

\author{
D.V. Solomatin \\ Omsk State Pedagogical University \\ *Corresponding Author: denis_2001j@bk.ru
}

Copyright $@ 2013$ Horizon Research Publishing All rights reserved.

\begin{abstract}
We study the proposed L.M. Martynov, the rank of a planarity for varieties of commutative semigroups.
\end{abstract}

Keywords Commutative Semigroups, Planarity, Cayley Graph

\section{Introduction}

Continuing the series of papers [1]-[5] on the proposed L.M.Martynovs Program for the Study of the planarity of Cayley graphs for varieties of semigroups, in this article we consideration not exposed earlier studies semigroup variety.

Recall that a variety $V$ of semigroups is ranked planarity $r$, where $r$ is the natural number, if all the $V$-free semigroup of rank $\leq r$ admits a planar Cayley graph, and $V$-free semigroup of rank $r+1$ does not allow planar Cayley graph. If for a variety $V$ of the natural number $r$ does not exist, then $V$ semigroups has infinite rank planarity [5]. Variety, each semigroup which admits a planar Cayley graph, called planar.

\section{Methodology}

Recall some definitions:

Definition 1. "Let $G$ be a semigroup, and let $S$ be a nonempty subset of $G$. The Cayley graph $\operatorname{Cay}(G, S)$ of $G$ relative to $S$ is defined as the graph with vertex set $G$ and edge set $E(S)$ consisting of those ordered pairs $(x, y)$ such that $s x=y$ for some $s \in S$ (see [6])"

Definition 2. "Planar graph is a graph that can be embedded in the plane, i.e., it can be drawn on the plane in such a way that its edges intersect only at their endpoints. In other words, it can be drawn in such a way that no edges cross each other. [7]"

Definition 3. "A semigroup is a set $S$ together with a binary operation "." (that is, a function $\cdot: S \times S \rightarrow S$ ) that satisfies the associative property: For all $a, b, c$ in $\mathrm{S}$, the equation $(a \cdot b) \cdot c=a \cdot(b \cdot c)$ holds. [8]"
Definition 4. "In mathematics, specifically universal algebra, a variety of algebras is the class of all algebraic structures of a given signature satisfying a given set of identities. Equivalently, a variety is a class of algebraic structures of the same signature that is closed under the taking of homomorphic images, subalgebras and (direct) products. In the context of category theory, a variety of algebras is usually called a finitary algebraic category.

Garrett Birkhoff proved equivalent the two definitions of variety given above, a result of fundamental importance to universal algebra and known as Birkhoff's theorem or as the HSP theorem. H, S, and P stand, respectively, for the closure operations of homomorphism, subalgebra, and product. [9]"

Definition 5. "In mathematics, a nowhere commutative semigroup is a semigroup $S$ such that, for all $a$ and $b$ in $S$, if $a b=b a$ then $a=b$. A semigroup $S$ is nowhere commutative if and only if any two elements of $S$ are inverses of each other. [10]"

Definition 6. "The free Burnside semigroup with $|A|$ generators defines by the equation $x^{n}=x^{n+m}$.[11]"

Definition 7. "A semigroup $S$ with zero for which there is an $n$ such that $S^{n}=0$; this is equivalent to the identity

$x_{1} \ldots x_{n}=y_{1} \ldots y_{n}$

in $S$. The smallest $n$ with this property for a given semigroup is called the step (sometimes class) of nilpotency. [12]"

Definition 8. The Abelian semigroups with exponents $p$ defines by the equations $x^{p+1}=x$ and $x^{p} y=y$. [12]

Definition 9. Commutative nil semigroups with step $n$ defines by the equations $x^{n+1}=x^{n}$ and $x^{n} y=x^{n}$. [12]

Definition 10. "A monoid is a set, $\mathrm{S}$, together with a binary operation "." (pronounced "dot" or "times") that satisfies the following three axioms:

Closure. For all $a, b$ in $S$, the result of the operation $a \cdot b$ is also in $S$.

Associativity. For all $a, b$ and $c$ in $S$, the equation $(a \cdot b) \cdot c=a \cdot(b \cdot c)$ holds.

Identity element. There exists an element $e$ in $S$, such that for all elements $a$ in $S$, the equation $e \cdot a=a \cdot e=a$ holds.

More compactly, a monoid is a semigroup with an identity element. It can also be thought of as a magma with 
associativity and identity. A monoid with invertibility property is a group. [13]"

Definition 11. "Complete bipartite graph on six vertices, three of which connect to each of the other three, also known as the utility graph, will be denoted by $K_{3,3}$ [14]"

\section{Results}

Let $r_{\pi}(\boldsymbol{V})$ - Rank planarity on the variety of commutative semigroups $\boldsymbol{V}$;

$\mathbf{C}=\operatorname{var}\{x y=y x\}-$ variety of all commutative semigroups;

$\mathbf{A}_{n}=\operatorname{var}\left\{x^{n} y=y, x y=y x\right\}$ - variety of Abelian semigroups with exponents $p>1$;

$\mathbf{C B}_{r, m}=\operatorname{var}\left\{x^{r+m}=x^{r}, x y=y x\right\}$ - variety of commutative burnsides semigroups, where $r>0, m>0$;

$\mathbf{C N}_{s}=\operatorname{var}\left\{x_{1} x_{2} \ldots x_{s} y=x_{1} x_{2} \ldots x_{s}, x y=y x\right\} \quad$ - variety of commutative nilpotent semigroup step $s>1$;

$\mathbf{C N i l}_{r}=\operatorname{var}\left\{x^{r} y=x^{r}, \quad x y=y x\right\}$ - variety of commutative nilsemigroups with index $r>1$;

then the main result is

Proposal. 1) $r_{\pi}(\mathbf{C})=2$;

2) $r_{\pi}\left(\mathbf{A}_{2}\right)=3$ and

$r_{\pi}\left(\mathbf{A}_{n}\right)=1$ with $n>2$;

3) $r_{\pi}\left(\mathbf{C B}_{1,1}\right)=3, r_{\pi}\left(\mathbf{C B}_{r, m}\right)=1$ with $r>0, m>2$, and

$r_{\pi}\left(\mathbf{C B}_{r, m}\right)=2$ in another cases;

4) $\quad r_{\pi}\left(\mathbf{C N}_{2}\right)=\infty, \quad r_{\pi}\left(\mathbf{C N}_{3}\right)=3 \quad$ and

$r_{\pi}\left(\mathbf{C N}_{\mathrm{s}}\right)=2$ with $s>3$;

5) $\quad r_{\pi}\left(\mathbf{C N i l}_{r}\right)=2$ with $r>1$.

Note that, by definition, trivial variety of single-element monoids has infinite rank and planarity is planar, in fact, a planar variety, obviously, are left zero semigroup variety and the variety of semigroups with zero multiplication.

\section{Discussion}

We also found another planar manifold defined by the identity properties $\left\{x y=x^{2} ; x y z=x y\right\}$, and formulated the hypothesis that the only listed semigroup variety planar.

Before developing the proposition of this hypothesis plausible reasoning: the basis of the Cayley graphs of semigroups varieties of left zero represent empty graph; basis of Cayley graphs of semigroups are a variety of combinations of steam, and Principles of Cayley graphs of semigroups with zero multiplication varieties are star, with the root at zero.

If there is a planar variety, which has a free semigroup Cayley graph, the base of which contains a vertex of which are branching down, by adding additional relationships with a sufficient number of generators can be obtained based on the graph Cayley subgraph homeomorphic to the graph $K_{3,3}$, that is, the diversity will not members of the planar, contradicting the choice of variety.

A rigorous proof of the corresponding statement is based on the machine computation and beyond the scope of this article, however, is true of the next

Theorem. The variety $\operatorname{var}\left\{x y=x^{2} ; x y z=x y\right\}$ is planar.

Proof. By definition, a planar manifold, each semigroup it must be planar. We outline the planar foundations of the Cayley graph of a free semigroup varieties $\operatorname{var}\left\{x y=x^{2} ; x y z=x y\right\}$ generated by the elements $\left\{a_{1}, a_{2}, \ldots, a_{n}\right\}$ in Fig. 1 .

According to Birkhoff's theorem, the manifold is closed under the operations of taking subsemigroups, and a homomorphic image of the direct product of semigroups. In this variety, these operations will maintain the planarity of the corresponding Cayley graphs.

The theorem is proved.

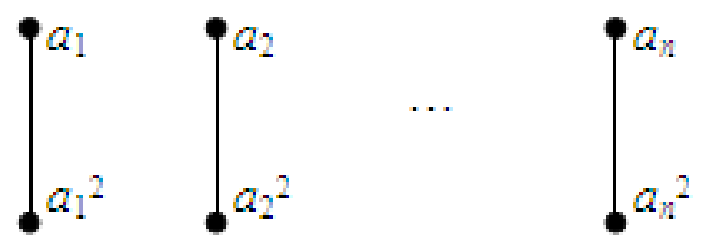

Figure 1. The scheme of planar foundation of the Cayley graphfree semigroup varieties $\operatorname{var}\left\{x y=x^{2} ; x y z=x y\right\}$

\section{Conclusion}

The author expresses his deep gratitude to Professor L.M. Martynov for in posing the problem, constant attention to this work and useful discussions.

\section{REFERENCES}

[1] Solomatin D.V., Finite free commutative semigroups with planar Cayley graphs, Mathematics and Computer Science: Science and Education, 3, OmSPU, Omsk, 2003, pp.32-38. (in Russian)

[2] Solomatin D.V., Finite free commutative monoids admitting a planar Cayley graphs, Communications of Omsk University, 4, OmSU, Omsk, 2005, pp.36-38. (in Russian)

[3] Solomatin D.V., Direct products of cyclic semigroups admitting a planar Cayley graph, Siberian Electronic Mathematical Reports, semr.math.nsc.ru, 3, 2006, pp.238-252. (in Russian)

[4] Solomatin D.V., Direct products of cyclic monoids and semigroups with zero admitting a planar Cayley graph, Mathematics and Computer Science: Science and Education: 6, OmSPU, Omsk, 2006, pp.51-63. (in Russian)

[5] Solomatin D.V., Planarity ranks of the varieties of commutative monoid / / Bulletin of Omsk university. HERALD OF OMSK UNIVERSITY. - Omsk State 
University Publishing House. (2012). - v. 4. (in Russian)

[6] Kelarev A.V., On undirected Cayley graphs Aust. J. Combin., 25 (2002), pp. 73-78

[7] Trudeau, Richard J. Introduction to Graph Theory (Corrected, enlarged republication. ed.). New York: Dover Pub. (1993) p. 64. ISBN 978-0-486-67870-2.

[8] Howie, John M., Fundamentals of Semigroup Theory, Clarendon Press, (1995) ISBN 0-19-851194-9.

[9] Banaschewski B., The Birkhoff Theorem for varieties of finite algebras, Algebra Universalis, Volume 17, Number 1 (1983), pp.360-368, DOI: 10.1007/ BF01194543

[10] Clifford A. H., Preston G. B. The Algebraic Theory of Semigroups Vol. I (Second Edition). American Mathematical
Society (1964), p.26. ISBN 978-0-8218-0272-4

[11] Alair Pereira do Lago, Maximal groups in free Burnside semigroups. LATIN'98: Theoretical Informatics Lecture Notes in Computer Science v.1380, (1998), pp 65-75

[12] Lyapin E.S., Semigroups, Amer. Math. Soc. (1974) (Translated from Russian)

[13] Howie, John M., Fundamentals of Semigroup Theory, London Mathematical Society Monographs. New Series 12, Oxford: Clarendon Press, (1995) ISBN 0-19-851194-9,Zbl 0835.20077

[14] Kuratowski Kazimierz, Sur le problème des courbes gauches en topologie, Fund. Math. (1930) v.15, pp.271-283. (in French) 